\title{
Epigenetic Markers in Perinatal Depression and Future Therapy
}

\author{
Jinghan Ruan
}

Bsc Psychology; University of Edinburgh; Edinburgh; United Kingdom; EH8 9AB
Corresponding author. Email: jinghanruan@gmail.com

\begin{abstract}
An increasing trend of perinatal depression was seen over the last decade and in both developed and underdeveloped regions. The purpose of this paper is to summarize the most predominant predictor of depressive symptoms during the vulnerable periods before and after pregnancy. For example, numerous epidemiological and case-control studies demonstrated that family and society play crucial roles in influencing mothers' mental states. More importantly, early life adversity such as childhood abuse, was indicated to increase the risk of depression by epigenetic embedding of inflammatory pathways. This biobehavioral process makes women more vulnerable during pregnancy and after giving birth. Antidepressant is an effective drug which was widely used to treat depression. Nevertheless, its exposure to infants during both prenatal and breastfeeding period is controlled for offspring's development. Therefore, psychological interventions such as IPT and CBT are more efficient and acceptable to reduce depressive symptoms for most pregnant women. Because PD is a multifactorial disorder, more studies are needed to enhance targeted interventions that can reduce the impact of $\mathrm{PD}$ on maternal-infant health.
\end{abstract}

Keywords: Epigenetics, perinatal depression, depression, pregnancy woman.

\section{INTRODUCTION}

Major Depressive Disorder (MDD) is one of the most prevalent mental disorders in 20th century, and it occurs more frequently among women than males. Additionally, experiencing pregnancy and birth delivering could increase the risk of suffering from major or minor depressive episodes. Perinatal depression (PD) is referred as a major depressive episode that occurs during pregnancy or within 4 months up to one year after child's birth [1]. Gavin et al. (2005) found that around $20 \%$ of females experience depressive symptoms during the perinatal period [2]. Moreover, most recent studies revealed that the global prevalence of antenatal depression was ranged from 15 to $65 \%$ [3]. Depression during prinatal period does not only impair mothers' mental health, but also affect children's cognitive, behavioral and psychological development later in life. For instance, it was demonstrated that offspring's stresscoping ability, emotion and temperament regulations were negatively impacted by PD through inheritance of genetic risk [4]. Consequently, PD has become an increasingly significant public health concern since its long-lasting and detrimental influence on both mothers and child development. However, most of the prior literatures were focused on the various adverse results that PD have made on fetus and infant's development. Therefore, the important predictors of PD were less researched, which is also considerably vital for enhancing public mental health and reducing females' unnecessary stress on pregnancy and parenting.

Over the past few decades, behavioral epigenetics is a new area of study in Psychology and has been a considerably dominant reason in explaining the exchange of phenotypes. It is defined as studies of how the environment and your behaviour could cause change to your genes with alternation of your DNA sequences [5]. There are three kinds of epigenetic changes that affect gene expression, which are DNA Methylation, Histone modification, and Non-coding RNA. The identification of associative biomarkers and epigenetic markers of PD could be helpful in developing new therapies. Therefore, to bring more insight and raise more awareness to females' mental health and the importance of PD. This paper is going to summarize and provide more insights of some of the most crucial impactors of developing PD from both environment and epigenetics by illustrating relevant studies, and give some suggestions to future treatments for PD. 


\section{ENVIRONMENTAL FACTORS}

\subsection{Demographic, socio-economic factors, and social support}

When considering the surrounding environment around a woman during pregnancy, family and partners are the most likely influencers that may induce the occurrences of PD. As expected, many previous studies have found relationships between them and perinatal depressive symptoms. For example, Ballenger conducted a large study in 2009 that allocated 40,333 participants around Australia [6]. They were asked to complete selfreport psychosocial risk factor questionnaire (PSRFQ), which included both demographic and psychosocial questions. Furthermore, mothers' postnatal depressive symptoms were measured by Edinburgh Postnatal Depression Scale (EPDS). The findings showed that inadequate partner support is one of the strongest predicting factors for PD. Even though this large cohort provided us significant, rich, and cross-sectional data and results, which demonstrating the powerful correlation between DP and demographic factors. Nevertheless, the use of self-report questionnaires and EPDS instead of clinical diagnosis was a limitation on data's accuracy. This finding was supported further by Ogbo et al.'s research in 2018, which also collected data from Australia but only included people from culturally and linguistically diverse (CALD) populations [7]. It was suggested that a lack of partner support is a risk factor for both prenatal and postnatal depressive symptoms. In addition to that, domestic or partner violence during pregnancy could also increase the likelihood of having PD [8].

Socio-economic level is also an important variable in psychological literatures, and a study in Goa, India (a low-income country) found a relationship between it and DP [9]. Patel et al. interviewed 270 pregnant mothers' demographic characteristics, such as age, education, socioeconomic status, marital violence, marital relationship etc. The results showed that poor marital relationship and economic deprivation are the crucial predisposing factors of $\mathrm{PD}$. This idea was also demonstrated by another meta-analysis in other countries. Nisar et al. selected 95 research studies and summarized that there was a significant inverse correlation between provincial Gross Domestic Product (GDP) and PD, which means areas with lower GDP had higher prevalence of PD in Mainland China [10]. Additionally, this review also indicated that reduced social support and poor demographic relationships were major influencers of perinatal depressive symptoms.

Apart from the risk factors that have been described previously, recent literatures also revealed a number of predisposing factors strongly associated with $\mathrm{PD}$, such as young age $[11,12]$, low income $[13,14]$, and low education attainment [11].

\subsection{Psychological and psychiatric factors}

Anxiety and depression were demonstrated to be highly comorbid, and many symptoms are overlapping [15]. Recent compelling evidence also supported an association between a history of anxiety and DP. Field et al. recruited 132 pregnant women who were middle socioeconomic status, and they were assigned to low anxiety and high anxiety groups by using a median split on the Trait Anxiety Scale [16]. After measuring their depression scores based on Center for Studies of Depression (CES-D), a positive correlation was indicated between anxiety and depression both prenatally and postnatally. Furthermore, a family history of mental illness is another example of psychiatric history which could impact the prevalence of PD [17]. Additionally, a follow-up study allocated 277 woman and their personality and clinical diagnosis of depression were assessed at 34 weeks pregnancy, and at 3, 6, and 12 months postpartum [18]. The results revealed that neuroticism and introversion are stable predictors of depressive symptoms in the first year after giving birth. What is more, another experiment by Dimitrovsky et al. accomplished this finding by demonstrating that there was a negative association between depression in pregnant woman and socially prescribed perfectionism [19].

\section{EPIGENETIC MECHANISM}

Many researches indicated that the socioeconomic disadvantage is positively associated with increased risk of depressive symptoms among women during pregnancy and after giving birth, such women are likely to experience more adverse life than higher-income ones. Therefore, early life event may be a possible factor which could exaggerate DP. Even though the studies on pregnant women are limited, this idea has been proved by embedding evidence. For example, women who underwent some traumatic events such as being abused at childhood were proposed to have higher probability of diagnosing postpartum depression [20]. Moreover, a most recent research proposed that pregnant women who had a history childhood sexual abuse had greater risk of having perinatal depressive and anxiety symptoms compared to those without such exposure [21]. The results showed that those symptoms reduced across the perinatal period, but they were highest during pregnancy. Thus, it is obvious that early life adversity (i.e., childhood abuse) is not only a major cause of MDD, but also a significant predictor of PD. However, the process of how early life adversity affects adult monoaminergic activity, and its etiology are remained unclear.

Recently, a hypothesis involved an active inflammatory process has become increasingly important in explaining the cause of MDD. Research indicated that activated macrophages produce proteins called proinflammatory cytokine in the upregulation of 
inflammatory reactions. The activation of inflammatory signaling pathways in the brain consequences in a lower serotonin level and higher glutamatergic effects [22, 23]. Furthermore, the proinflammatory cytokine could also play a predominant role in mediating between both genetic and environmental predictors which elevates the development of MDD. Additionally, embedding evidence have applied the theory of inflammation to PD [24]. Therefore, Garfield et al. came up with a hypothesis to explain the epigenetic mechanism between early life adversity and PD based on the inflammatory theory of depression and biological embedding of childhood adversity model [25]. The early life adversity-perinatal depressive risk model suggested that mothers' early poor experience embeds an epigenetic proinflammatory signature which imprints long-term epigenetic alternations (e.g., DNA methylation) in responding to stressors related to pregnancy and child parenting. As a result, decreased levels of oxytocin and higher levels of proinflammatory cytokines promote a maternal proinflammatory phenotype and thus increase the risk for inflammation-related maternal depressive symptoms. Thus, early traumatic experience is one of the predominant factors that relate to $\mathrm{PD}$ and/or predict the development of PD.

Over the past few decades, DNA methylation has been one of the main epigenetic mechanisms which were studied most frequently. It is a biological process of adding a chemical group to DNA without changing the sequence. Additionally, it also plays a key role in early life adversity induced mental illness [26]. To find out the overlap in patterns DNA methylation in correlation with PD, childhood adversity, and insecure attachment style, Robakis et al. [27] conducted a longitudinal prospective study with 54 women having PD. This large-scale of analysis identified 3 regions in which methylation density was associated with insecure attachment style, and 6 in which methylation density was associated with PD, 1580 in which methylation density was associated with early life adversity. Furthermore, identical patterns of DNA methylation in association with PD and with insecure attachment style were observed in a detailed analysis of methylation density in the oxytocin receptor gene. Nevertheless, childhood trauma was associated with a distinct methylation pattern in this particular gene. Consequently, PD is a multifactorial disorder, and it is necessary to further investigate the biobehavioral mechanism for PD in order to maintain a better understanding. Therefore, the identification of vulnerable woman and therapy interventions could be modified and improved.

\section{FUTURE THERAPY}

Given the increasing prevalence of PD and its adverse effects on both of the mothers' mental illness and infants' cognitive abilities, PD's depressive symptoms need to be identified promptly. Furthermore, the intervention of treatments for this mental illness has important public health implications. Nowadays, antidepressant is one of the most effective and frequently used methods to treat depression. Nevertheless, if women consume drugs during pregnancy, fetus' development could be largely retarded depends on their exposure to the antidepressants. Therefore, medication dosage is limited for pregnant woman whereas the majority of them require higher level of dosage to reduce depressive symptoms during perinatal period [28]. Moreover, this problem is also occurred after mothers giving birth. For instance, lactating women are prescribed with lower dosage level of antidepressants to treat PD because antidepressants could be absorbed by infants through breast-feeding.

Apart from antidepressant medication, the psychotherapeutic intervention is another most common therapy. Additionally, many surveys have analyzed that the majority of mothers who are pregnant or breastfeeding have a higher acceptability for psychological treatment than pharmacological treatment during both pregnancy and the postnatal period [29]. A systemic meta-analysis was conducted to examine the efficacy of psychological and pharmacologic interventions for treatment of PD. The selected 27 studies included different types of therapies. For instance, the Interpersonal psychotherapy (IPT) was the most prevalent psychological intervention, followed by cognitive behavioral therapy (CBT), and etc. Furthermore, the target population, intervention location and types were also reported and analyzed. The findings showed that both of the IPT and CBT were correlated with a greater reduction of depressive symptoms from pretest to posttreatment. Interestingly, it also found that IPT reported higher effect sizes when compared to controls and was superior to CBT. Linking it back to the predisposing factors which have been identified previously, psychological interventions of therapies could have better outcomes on pregnant women who had problems in interpersonal relationships or life experience. Consequently, the results had vital implications for both researchers and clinicians, and assessments of sociodemography, childhood adversity, personality, recent life events and psychiatric history should be included more explicitly in both the evaluation and treatment planning for PD patients. Moreover, individual psychotherapy was demonstrated to be more superior than group psychotherapy when observing changes in symptoms [30].

An individually-administered therapeutic interventions of method may be more efficient and suitable than uniformed preset treatments because individuals have distinct combinations of causal factors of PD. However, relatively few researches of antidepressant medication have been conducted among this population. Therefore, more studies are needed to confirm further the advantages of psychological therapies and test the effectiveness of methods combing both 
antidepressant medication and psychological interventions for treating PD.

\section{CONCLUSION}

Results of these researches and analyses provide evidence for confirming an increasing trend of PD. Moreover, its negative consequences on mothers' mental health could further influence offspring's cognitive and emotional development. Prior literatures have identified that PD is caused by multifactorial disorder, and some predominant contributors have been investigated a lot. However, a majority of those studies were conducted in low-income countries and with very limited sample size. Thus, more epidemiological research on wider range of population in less developed regions are needed to identify the influencers that have not been identified yet. Epigenetics mechanism is another explanation of PD, this biological process elevates the development by associating the environmental predictors with genes. Nevertheless, this finding needs confirmation from more replications and future studies.

Therefore, various support programs including psychosocial interventions are needed to eliminate the development of possible risky factors. For instance, parenting education could help reduce their stress before giving birth. Moreover, adequate support from society and family is important since most cases of PD was elevated by lack of understanding and assistant from surroundings. To diminish early life social experience and adversity, reversed lifestyle interventions have been introduced recently. Methods such as increasing physical activities, reducing psychological stress, enriching early life experiences, and modifying diet are identified as effective. Additionally, many researches have found that psychological therapies such as IPT and CBT are the most efficient treatment for PD. However, because there are various contributors of this mental illness and patients' situations may differ with one another. Thus, a specialized program of individual treatment combining both psychosocial intervention and optimal antidepressant medication dosage may be the most effective method. In conclusion, urgent attention is required to address this public health priority, and more research is needed to help modify prevention, identification and acceptable treatments for PD.

\section{ACKNOWLEDGMENTS}

This paper would not have been possible without the exceptional support of my supervisor, Susan. Her generous assistant, and knowledge have been an inspiration and kept my work on track.

\section{REFERENCES}

[1] Dagher, R. K., Bruckheim, H. E., Colpe, L. J., Edwards, E., \& White, D. B. (2021). Perinatal
Depression: Challenges and Opportunities. Journal of Women's Health, 30(2), 154-159. https://doi.org/10.1089/jwh.2020.8862

[2] Gavin, N. I., Gaynes, B. N., Lohr, K. N., MeltzerBrody, S., Gartlehner, G., \& Swinson, T. (2005). Perinatal Depression: a systematic review of prevalence and incidence. Obstetrics \& Gynecology, 106(5, Part 1), 1071-1083. https://doi.org/10.1097/01.aog.0000183597.31630. $\mathrm{db}$

[3] Dadi, A. F., Miller, E. R., Bisetegn, T. A., \& Mwanri, L. (2020). Global burden of antenatal depression and its association with adverse birth outcomes: an umbrella review. BMC Public Health, 20(1). https://doi.org/10.1186/s12889-020-8293-9

[4] Waxler, E., Thelen, K., \& Muzik, M. (2011). Maternal perinatal depression-impact on infant and child development. Eur Psychiatr Rev, 4(1), 41-47.

[5] Berger, S. L., Kouzarides, T., Shiekhattar, R., \& Shilatifard, A. (2009). An operational definition of epigenetics. Genes \& Development, 23(7), 781783. https://doi.org/10.1101/gad.1787609

[6] Ballenger, J. C. (2009). Antenatal risk factors for postnatal depression: A large prospective study. Yearbook of Psychiatry and Applied Mental Health, 2009, 202-203. https://doi.org/10.1016/s00843970(08)79169-2

[7] Ogbo, F. A., Eastwood, J., Hendry, A., Jalaludin, B., Agho, K. E., Barnett, B., \& Page, A. (2018). Determinants of antenatal depression and postnatal depression in Australia. BMC Psychiatry, 18(1). https://doi.org/10.1186/s12888-018-1598-X

[8] Edwards, B., Galletly, C., Semmler-Booth, T., \& Dekker, G. (2008). Antenatal psychosocial risk factors and depression among women living in socioeconomically disadvantaged suburbs in Adelaide, South Australia. Australian \& New Zealand Journal of Psychiatry, 42(1), 45-50. https://doi.org/10.1080/00048670701732673

[9] Patel, V., Rodrigues, M., \& DeSouza, N. (2002). Gender, poverty, and Postnatal Depression: A study of mothers in Goa, India. American Journal of Psychiatry, 159(1), 43-47. https://doi.org/10.1176/appi.ajp.159.1.43

[10] Nisar, A., Yin, J., Waqas, A., Bai, X., Wang, D., Rahman, A., \& Li, X. (2020). Prevalence of perinatal depression and Its determinants in Mainland China: A systematic review and metaanalysis. Journal of Affective Disorders, 277, 1022 1037. https://doi.org/10.1016/j.jad.2020.07.046 
[11] Marcus, S. M., Flynn, H. A., Blow, F. C., \& Barry, K. L. (2003). Depressive symptoms among pregnant women screened in obstetrics settings. Journal of Women's Health, 12(4), 373-380. https://doi.org/10.1089/154099903765448880

[12] Rubertsson, C., Waldenström, U., \& Wickberg, B. (2003). Depressive mood in Early Pregnancy: Prevalence and women at risk in a national Swedish sample. Journal of Reproductive and Infant Psychology, 21(2), 113-123. https://doi.org/10.1080/0264683031000124073

[13] Woody, C. A., Ferrari, A. J., Siskind, D. J., Whiteford, H. A., \& Harris, M. G. (2017). A systematic review and meta-regression of the prevalence and incidence of perinatal depression. Journal of Affective Disorders, 219, 86-92. https://doi.org/10.1016/j.jad.2017.05.003

[14] Bolton, H. L., Hughes, P. M., Turton, P., \& Sedgwick, P. (1998). Incidence and demographic correlates of depressive symptoms during pregnancy in an inner London population. Journal of Psychosomatic Obstetrics \& Gynecology, 19(4), 202-209. https://doi.org/10.3109/01674829809025698

[15] Gorman, J. M. (1996). Comorbid depression and ANXIETY spectrum disorders. Depression and Anxiety, 4(4), 160-168. https://doi.org/10.1002/(sici)15206394(1996)4:4<160::aid-da2>3.0.co;2-j

[16] Field, T., Diego, M., Hernandez-Reif, M., Schanberg, S., Kuhn, C., Yando, R., \& Bendell, D. (2003). Pregnancy anxiety and comorbid depression and anger: Effects on the fetus and neonate. Depression and Anxiety, 17(3), 140-151. https://doi.org/10.1002/da.10071

[17] Johnstone, S. J., Boyce, P. M., Hickey, A. R., Morris-Yates, A. D., \& Harris, M. G. (2001). Obstetric risk factors for postnatal depression in urban and rural community samples. Australian \& New Zealand Journal of Psychiatry, 35(1), 69-74. https://doi.org/10.1046/j.1440-1614.2001.00862.x

[18] Verkerk, G. J. M., Denollet, J., Van Heck, G. L., Van Son, M. J. M., \& Pop, V. J. M. (2005). Personality factors as determinants of depression in postpartum women: A prospective 1-year follow-up study. Psychosomatic Medicine, 67(4), 632-637. https://doi.org/10.1097/01.psy.0000170832.14718. 98

[19] Dimitrovsky, L., Levy-Shiff, R., \& SchattnerZanany, I. (2002). Dimensions of depression and perfectionism in pregnant and Nonpregnant WOMEN: Their levels and
INTERRELATIONSHIPS and their relationship to marital satisfaction. The Journal of Psychology, 136(6), 631-646. https://doi.org/10.1080/00223980209604824

[20] Buist, A. (1998). Childhood abuse, postpartum depression and parenting difficulties: A literature review of associations. Australian \& New Zealand Journal of Psychiatry, 32(3), 370-378. https://doi.org/10.3109/00048679809065529

[21] Akinbode, T. D., Pedersen, C., \& Lara-Cinisomo, S. (2020). The price of pre-adolescent abuse: Effects of sexual abuse on perinatal depression and anxiety. Maternal and Child Health Journal, 25(7), 10831093. https://doi.org/10.1007/s10995-020-03088-X

[22] Danese, A., Moffitt, T. E., Pariante, C. M., Ambler, A., Poulton, R., \& Caspi, A. (2008). Elevated inflammation levels in depressed adults with a history of childhood maltreatment. Archives of General Psychiatry, 65(4), 409. https://doi.org/10.1001/archpsyc.65.4.409

[23] Oxenkrug, G. (2013). Serotonin - kynurenine hypothesis of Depression: Historical overview and recent developments. Current Drug Targets, 14(5), 514-521. https://doi.org/10.2174/1389450111314050002

[24] Anderson, G., \& Maes, M. (2013). Postpartum depression: Psychoneuroimmunological underpinnings and treatment. Neuropsychiatric Disease and Treatment, 277. https://doi.org/10.2147/ndt.s25320

[25] Garfield, L., Mathews, H. L., \& Janusek, L. W. (2015). Inflammatory and epigenetic pathways for perinatal depression. Biological Research For Nursing, 18(3), 331-343. https://doi.org/10.1177/1099800415614892

[26] Xu, Q., Jiang, M., Gu, S., Wang, F., \& Yuan, B. (2020). Early life stress induced dna methylation of monoamine oxidases leads to depressive-like behavior. Frontiers in Cell and Developmental Biology, 8. https://doi.org/10.3389/fcell.2020.582247

[27] Robakis, T. K., Zhang, S., Rasgon, N. L., Li, T., Wang, T., Roth, M. C., Humphreys, K. L., Gotlib, I. H., Ho, M., Khechaduri, A., Watson, K., RoatShumway, S., Budhan, V. V., Davis, K. N., Crowe, S. D., Ellie Williams, K., \& Urban, A. E. (2020). Epigenetic signatures of attachment insecurity and childhood adversity provide evidence for role transition in the pathogenesis of perinatal depression. Translational Psychiatry, 10(1). https://doi.org/10.1038/s41398-020-0703-3 
[28] Bennett, H. A., Einarson, A., Taddio, A., Koren, G., \& Einarson, T. R. (2004). Depression during pregnancy. Clinical Drug Investigation, 24(3), 157179. https://doi.org/10.2165/00044011-20042403000004

[29] Kim, D. R., Sockol, L., Barber, J. P., Moseley, M., Lamprou, L., Rickels, K., O'Reardon, J. P., \& Epperson, C. N. (2011). A survey of patient acceptability of repetitive transcranial magnetic stimulation (tms) during pregnancy. Journal of Affective Disorders, 129(1-3), 385-390. https://doi.org/10.1016/j.jad.2010.08.027

[30] Sockol, L. E., Epperson, C. N., \& Barber, J. P. (2011). A meta-analysis of treatments for perinatal depression. Clinical Psychology Review, 31(5), 839-849. https://doi.org/10.1016/j.cpr.2011.03.009

[31] Ritter, C., Hobfoll, S. E., Lavin, J., Cameron, R. P., \& Hulsizer, M. R. (2000). Stress, psychosocial resources, and depressive symptomatology during pregnancy in low-income, inner-city women. Health Psychology, 19(6), 576-585. https://doi.org/10.1037/0278-6133.19.6.576 